PHYSICAL REVIEW D 96, 101302(R) (2017)

\title{
Averaged energy conditions and bouncing universes
}

\author{
Massimo Giovannini \\ Department of Physics, Theory Division, CERN, 1211 Geneva 23, Switzerland \\ INFN, Section of Milan-Bicocca, 20126 Milan, Italy \\ (Received 29 August 2017; published 16 November 2017)
}

\begin{abstract}
The dynamics of bouncing universes is characterized by violating certain coordinate-invariant restrictions on the total energy-momentum tensor, customarily referred to as energy conditions. Although there could be epochs in which the null energy condition is locally violated, it may perhaps be enforced in an averaged sense. Explicit examples of this possibility are investigated in different frameworks.
\end{abstract}

DOI: 10.1103/PhysRevD.96.101302

Since the first WMAP releases, the upper limits on the tensor-to-scalar ratio $r_{T}$ have been steadily decreasing so that it is reasonable to admit, with a fair degree of confidence, that $r_{T}$ must not exceed $1 / 10$ at a typical pivot scale of $0.002 \mathrm{Mpc}^{-1}$ [1]. The conventional inflationary scenarios (broadly compatible with the latter upper limit) would imply a set of Cauchy data characterized by a minute energy density of the inflaton in Planck units [1]. The current bounds cannot exclude even smaller values of $r_{T}$ (e.g., of the order of $10^{-5}$ ). Since bouncing universes naturally lead to extremely small tensor-to-scalar ratios [2], they might represent a plausible completion of the protoinflationary dynamics (see, for instance, Ref. [3] for an incomplete list of reviews involving, directly or indirectly, bouncing scenarios).

Bouncing universes are sometimes ruled out (or in) by appealing to various coordinate-invariant restrictions on the total energy-momentum tensor $T_{\mu \nu}$. These restrictions are conventionally referred to as energy conditions [3,4]. In various classes of theories, bouncing solutions violate the null energy condition, ${ }^{1}$ demanding that $T_{\mu \nu} k^{\mu} k^{\nu} \geq 0$, where $k^{\mu}$ is a null vector (i.e., $g_{\mu \nu} k^{\mu} k^{\nu}=0$, and $g_{\mu \nu}$ denotes the four-dimensional metric tensor). Whenever the null energy condition is strictly enforced, the occurrence of bouncing universes is prevented, at least in the scenarios formulated within the conventional general relativistic dynamics. Indeed, if this is the case, the first (cosmic) time derivative

\footnotetext{
*massimo.giovannini@cern.ch

${ }^{1}$ The contraction of arbitrary timelike or null vectors with $T_{\mu \nu}$ leads to a number of different scalar functions playing a relevant role in various singularity theorems [4]. Besides the null energy condition, the weak energy condition stipulates that $T_{\mu \nu} q^{\mu} q^{\nu} \geq 0$, where $q^{\mu}$ is a timelike vector (i.e., $q^{\mu} q^{\nu} g_{\mu \nu}=1$ ). Conversely, the strong energy condition implies that $T_{\mu \nu} p^{\mu} p^{\nu} \geq T_{\lambda}^{\lambda} p^{\sigma} p_{\sigma} / 2$, where $p^{\mu}$ is now a nonspacelike vector (i.e., either timelike or null).
}

Published by the American Physical Society under the terms of the Creative Commons Attribution 4.0 International license. Further distribution of this work must maintain attribution to the author(s) and the published article's title, journal citation, and DOI. of the Hubble rate is always negative semidefinite and cannot change sign. The same kind of restrictions appear when applying effective field theory methods to the analysis of single-field cosmological models in which the dependence of the action on the scalar field can be constrained through the energy conditions. The effective field theory approach (originally developed in the framework of single-field inflationary scenarios [5]) can be translated into the case of bouncing dynamics, and in this context, the enforcement of the null energy condition has been used to rule out a large class of realizations of effective bouncing scenarios [6]. The question we ought to address in this paper is the following: even if the null energy condition is violated locally in a given bouncing universe, can it be satisfied on average? While there is no general proof of this statement, we shall show that the answer can be affirmative at least in a few explicit examples.

Although there are regions where the null energy condition can be locally violated, it may perhaps be satisfied in some averaged sense; this is, in a nutshell, the conservative suggestion conveyed hereunder in connection with the bouncing dynamics. The notion of averaged energy conditions (originally introduced by Ford and subsequently sharpened in a series of papers [7]) aims at preventing the violation of the second law of thermodynamics and also plays a relevant role in the derivation of the so-called quantum interest conjecture. So far, averaged energy conditions have not been specifically analyzed in the context of bouncing scenarios; the goal of the present investigation is to fill this gap, at least partially. For the present goals, a direct way of introducing the averaged null energy condition is to consider the integral [7]

$$
\int_{\gamma} T_{\mu \nu} k^{\mu} k^{\nu} d \lambda \geq 0
$$

where $k^{\mu}=d x^{\mu} / d \lambda$ is the tangent vector to the null geodesic and $\lambda$ is an affine parameter with respect to which the tangent vector to the geodesic is defined; in Eq. (1), $\gamma$ denotes a null geodesic. In general relativity, the integral condition (1) can also be phrased by substituting $T_{\mu \nu}$ with 
the Ricci tensor $R_{\mu \nu}$; the two conditions are in fact equivalent (up to numerical factors) by definition of a null vector and thanks to the field equations. ${ }^{2}$

To assess the restrictions implied by Eq. (1), the case of conformally flat Friedmann-Robertson-Walker geometries shall be preferentially considered, but the possible extensions of this study to open or closed universes seem very plausible; the metric tensor shall then be expressed, for the present ends, $\operatorname{as}^{3} g_{\mu \nu}=a^{2}(\tau) \eta_{\mu \nu}$, where $\eta_{\mu \nu}=\operatorname{diag}(1,-1$, $-1,-1)$ is the Minkowski metric and $a(\tau)$ is the scale factor in the conformal time parametrization. In the case of a conformally flat metric, the null vector $k^{\mu}$ can always be parametrized as $k^{\mu}=a^{-2}\left(1, n^{i}\right)$, where $n_{i} n^{i}=1$. In this way, $k^{\mu}$ coincides with the tangent to an affinely parametrized geodesic, and Eq. (1) can be written as

$$
\int_{\gamma} T_{\mu \nu} k^{\mu} k^{\nu} d \lambda=\int_{\gamma}\left(p_{t}+\rho_{t}\right) d \tau \geq 0
$$

where $\left(p_{t}+\rho_{t}\right)$ denotes the total enthalpy density of the system and the equality follows by recalling that, in the present parametrization of null vectors $k^{\mu}, d \tau / d \lambda=a^{-2}$. If the null energy condition is enforced for each and every value of the conformal time coordinate, the bouncing dynamics is prevented. We shall therefore be concerned with the situation in which the null energy condition is violated in a limited range of the conformal time coordinate $\tau$ and, more specifically, between $-\tau_{-}$and $\tau_{+}$. Indeed, diverse solutions often employed to construct phenomenological scenarios can be divided into three distinct epochs: besides the intermediate time range (i.e., $-\tau_{-}<\tau<\tau_{+}$) in which the null energy condition is either partially or completely violated, one can define two asymptotic regions (respectively, for $\tau<-\tau_{-}$and for $\tau>\tau_{+}$) where the null, dominant, and weak energy conditions are enforced. ${ }^{4}$ With

\footnotetext{
${ }^{2}$ The averaged null energy condition measures the degree of violation of the null energy condition. It has been argued (see, e.g., the second paper of Ref. [7]) that various general relativistic results can be demonstrated without requiring that energy conditions are satisfied locally, but only in an averaged sense.

${ }^{3} \mathrm{We}$ are dealing here with the case in which the spatial curvature is absent (this is an immediate consequence of conformal flatness). In the presence of spatial curvature, the bouncing behavior may arise without an explicit violation of the null energy condition, as in the case of the de Sitter bounce, which may also arise when the total energy-momentum tensor is dominated by a scalar field with specific potential. It has been well known since the early 1970s that bouncing models may arise in the presence of spatial curvature without an explicit violation of the null energy condition [8], but this is not the situation explored in this paper.

${ }^{4}$ In spite of the conservative viewpoint of this discussion, it could also be plausible to analyze, within the present framework, all those scenarios in which the violation of the null energy condition occurs for $\tau \rightarrow-\infty$ (see, e.g., the last paper of Ref. [6] for some examples along this direction); these potentially interesting generalizations, however, shall not be discussed here.
}

these specifications, the restrictions implied by Eq. (2) can be rephrased as

$$
\left|\int_{-\tau_{-}}^{\tau_{+}}\left(p_{t}+\rho_{t}\right) d \tau\right| \leq \int_{-\infty}^{-\tau_{-}}\left(p_{t}+\rho_{t}\right) d \tau+\int_{\tau_{+}}^{+\infty}\left(p_{t}+\rho_{t}\right) d \tau,
$$

where, by definition, $\left(p_{t}+\rho_{t}\right)_{\tau_{ \pm}} \rightarrow 0$, while it is negative for $-\tau_{-}<\tau<\tau_{+}$. Equation (3) assumes that the curvature invariants are all regular and that the causal (i.e., the nonspacelike) geodesics are all complete and extendible to arbitrary values of their affine parameter.

Since what matters is the total enthalpy density, Eqs. (2) and (3) do not solely depend on the dynamics of the background but also on the amplified inhomogeneities and, in particular, on those species that are not conformally coupled to the geometry. More specifically, if $N_{n c}$ is the number of nonconformally coupled species, it is well known that gravitational particle production at the end of inflation roughly contributes to the total energy density as $\mathcal{N}_{n c} H_{f}^{4}\left(a_{f} / a\right)^{4}$, where $H_{f}$ is the Hubble rate at the end of inflation [9] and $\mathcal{N}_{n c}=N_{n c} \zeta_{n c}$; the numerical factor $\zeta_{n c}<1$ may change, in principle, from species to species. Following earlier and more recent estimates [9], we can have that $\zeta_{n c}=\mathcal{O}(0.01)$ for minimally coupled scalars so that, without loss of generality, we shall assume $\mathcal{N}_{n c}=N_{n c} / 100$. Similar values of $\zeta_{n c}$ arise when computing the total energy density of gravitons since the two tensor polarizations (evolving in a conformally flat Friedmann-Robertson-Walker metric) obey the evolution of two independent scalar fields minimally coupled to the geometry [9]. While the specific values of $\zeta_{n c}$ will be largely immaterial for the present purposes, the observations of Refs. [9,10] (see also first paper of Ref. [11]) cannot be disregarded whenever the rate of asymptotic expansion for $\tau \gg \tau_{+}$is smaller than the expansion rate of radiation. ${ }^{5}$ In the latter case, the energy density of the nonconformally coupled quanta may even become asymptotically dominant [9]. For the sake of concreteness, we shall therefore assume that in the limits $\tau \rightarrow \mp \infty$ the background scale factors evolve, respectively, as

$\lim _{\tau \rightarrow-\infty} a(\tau) \rightarrow a_{-}\left(-\frac{\tau}{\tau_{-}}\right)^{\alpha_{-} / 2}, \quad \lim _{\tau \rightarrow+\infty} a(\tau) \rightarrow a_{+}\left(\frac{\tau}{\tau_{+}}\right)^{\alpha_{+} / 2}$.

While the $\tau \rightarrow-\infty$ limit of the solution will only be determined by the evolution of the background, in the opposite limit, the role of the produced massless quanta will

\footnotetext{
${ }^{5}$ It is interesting to remark that this observation has been used even prior to the formulation of the inflationary hypothesis; in a specific toy model, Parker demonstrated that the backreaction of massless quanta may lead to a thermal background radiation [10].
} 
depend on the value of $\alpha_{+}$; the total energy density for $\tau>\tau_{+}$will be in fact given by

$$
\rho_{t}=\frac{3 H_{+}^{2} M_{P}^{2}}{8 \pi}\left(\frac{a_{+}}{a}\right)^{2+4 / \alpha_{+}}+\mathcal{N}_{n c} H_{+}^{4}\left(\frac{a_{+}}{a}\right)^{4} .
$$

Note that $\mathcal{N}_{n c} \geq 1 / 50$ (where the equality holds if we just consider the two tensor polarizations). According to the parametrization of Eq. (5), if $\alpha_{+}<2$, the expansion rate will be slower than in the case of radiation; conversely, if $\alpha_{+} \geq 2$, the asymptotic rate of expansion will either be comparable to or larger than the one typical of a radiationdominated phase. This means that when $\alpha_{+}<2$ the asymptotic contribution of the massless species will dominate; more specifically, this will be the case for $a>a_{*}$, where $a_{+} / a_{*}$ is defined as

$$
\frac{a_{+}}{a_{*}}=\left(\frac{8 \pi \mathcal{N}_{n c}}{3}\right)^{\alpha_{+} /\left(4-2 \alpha_{+}\right)}\left(\frac{H_{+}}{M_{P}}\right)^{\alpha_{+} /\left(2-\alpha_{+}\right)} .
$$

In the opposite dynamical regime (i.e., $\alpha_{+} \geq 2$ ), the backreaction of the massless quanta is still conceptually relevant but does not have appreciable physical effects on the evolution of the background.

If $\alpha_{+}<2$, the effect of the second term on the right-hand side of Eq. (5) dominates the energy density for typical time scales $\tau_{*}=\mathcal{O}\left(\tau_{+}\right)$whenever $H_{+}=\mathcal{O}\left(M_{P}\right)$. Conversely, the dominance of the amplified quantum fluctuations will take place on a time scale much larger than the typical duration of the bouncing regime (i.e., $\tau_{*} \gg \tau_{+}$whenever $\left.H_{+} / M_{P} \ll 1\right)$. For the sake of definiteness, we can consider the standard Friedmann-Lemaittre equations

$$
\begin{aligned}
3 M_{P}^{2} \mathcal{H}^{2} & =8 \pi \rho_{b} a^{2}, \\
M_{P}^{2}\left(\mathcal{H}^{2}-\mathcal{H}^{\prime}\right) & =4 \pi\left(\rho_{b}+p_{b}\right) a^{2},
\end{aligned}
$$

where $M_{P}=1 / \sqrt{G}$ denotes the Planck mass; a particular solution of Eq. (7) compatible with a local violation of the null energy condition and expanding at a rate slower than radiation for $\tau>\tau_{+}$is obtained in terms of the scale factor,

$$
a(\tau)=a_{1}\left(x^{2}+1\right)^{1 / 4}, \quad x=\tau / \tau_{1},
$$

leading, through Eq. (7), to the following energy and enthalpy densities ${ }^{6}$ :

$$
\begin{aligned}
\rho_{b} & =\frac{3 M_{P}^{2}}{32 \pi a_{1}^{2} \tau_{1}^{2}} \frac{x^{2}}{\left(x^{2}+1\right)^{5 / 2}}, \\
\rho_{b}+p_{b} & =\frac{M_{P}^{2}}{16 \pi a_{1}^{2} \tau_{1}^{2}} \frac{\left[3 x^{2}-2\right]}{\left(x^{2}+1\right)^{5 / 2}} .
\end{aligned}
$$

\footnotetext{
${ }^{6}$ Note that the condition (1) is not bound to hold in flat space. It is however true that the background models examined in this paper all have flat space-time as asymptotic limit for $\tau \rightarrow-\infty$.
}

Equation (8) describes an accelerated contraction for $\tau<-\tau_{-}$turning into a decelerated expansion for $\tau>\tau_{+}$. Equation (9) implies $\tau_{+}=\tau_{-}=\sqrt{2 / 3} \tau_{1}$, while $\alpha_{+}=$ $\alpha_{-}=1$. In the example of Eq. (8), the energy density of the nonconformally coupled species can be directly computed by studying the evolution equation of the corresponding mode functions. The production of massless quanta is governed (up to a numerical factor determining $\left.\zeta_{n c}\right)$ by $a^{\prime \prime} / a[9,11]$. This function can be explicitly computed, for instance, in the case of Eq. (8), and it can be verified that all the Fourier modes $k<\mathcal{O}\left(1 / \tau_{1}\right)$ will indeed be amplified up to a critical wave number ${ }^{7}$ $k_{\max }=\mathcal{O}\left(a_{1} H_{1}\right)$. The energy density of the produced quanta will then be dominated by the largest amplified mode, and this is why it is of the order of $k_{\max }^{4} \simeq H_{1}^{4} a_{1}^{4}$ [9].

Owing to the results of Eqs. (5), (8), and (9), the explicit form of Eq. (2) becomes

$$
\begin{aligned}
\int_{-\infty}^{+\infty}\left(p_{t}+\rho_{t}\right) d \tau= & \frac{M_{P} H_{1}^{2}}{16 \pi \tau_{1} a_{1}^{2}}\left[\int_{-\infty}^{\tau_{*} / \tau_{1}} \frac{3 x^{2}-2}{\left(x^{2}+1\right)^{5 / 2}} d x\right. \\
& \left.+\frac{64 \pi}{9} \mathcal{N}_{n c}\left(\frac{H_{*}}{H_{1}}\right)\left(\frac{H_{*}}{M_{P}}\right)^{2}\left(\frac{a_{1}}{a_{*}}\right)\right] .
\end{aligned}
$$

If we now recall that $H_{1}=\mathcal{H}_{1} / a_{1}$, we can also deduce, from Eqs. (5) and (8), the following chain of equalities,

$$
\left(\frac{a_{1}}{a_{*}}\right)^{2}=\frac{\tau_{1}}{\tau_{*}}=\frac{8 \pi}{3} \mathcal{N}_{n c}\left(\frac{H_{1}}{M_{P}}\right)=\epsilon,
$$

where, for the sake of conciseness, we introduced the dimensionless parameter $\epsilon$. Using Eq. (11), the result of Eq. (10) can also be expressed as

$$
\int_{-\infty}^{+\infty}\left(p_{t}+\rho_{t}\right) d \tau=\frac{H_{1}^{2}}{\tau_{1} a_{1}^{2}}\left[\int_{-\infty}^{1 / \epsilon} \frac{3 x^{2}-2}{\left(x^{2}+1\right)^{5 / 2}} d x+\frac{8}{3} \epsilon^{6}\right]
$$

to get rid of the $M_{P}^{2} /(16 \pi)$ in the prefactor of Eq. (12), we adopted units $16 \pi G=1$. Equation (12) can be used within two complementary approaches, which are, in short, the following. In a given bouncing scenario and for a specific value of $\mathcal{N}_{c}$, the value of $\epsilon$ can be accurately assessed; this analysis will show if (and how) the (averaged) null energy condition is enforced. Conversely, in a more heuristic perspective, we can use the positivity of the averaged null energy condition to restrict the values of $\epsilon$. According to this second strategy, the integral appearing in Eq. (12) can be performed analytically; the null energy condition is positive (on average), provided $0.8<\epsilon<1$, implying that $H_{1} \leq \mathcal{O}\left(M_{P}\right)$. Thus, by enforcing the averaged null energy condition, the maximal scale of the bounce can be

\footnotetext{
${ }^{7}$ We recall that, as usual, $\mathcal{H}=a H$, where $H$ denotes the conventional Hubble rate.
} 
MASSIMO GIOVANNINI

determined. Absent any contribution from the nonconformally coupled species, the integrals on the right-hand side of Eq. (3) are subdominant if compared with the integral on the left-hand side. However, when the backreaction effects are taken into account, the second integral on the right-hand side of Eq. (3) becomes larger than the first contribution, and this implies that the averaged null energy condition is not violated, provided $H_{1}=\mathcal{O}\left(M_{P}\right)$.

All in all, we can say that, as long as $H_{1} \ll M_{P}$, the contribution of the nonconformally coupled species cannot restore the validity of the averaged null energy condition. ${ }^{8}$ The opposite is true in the case in which $H_{1}=\mathcal{O}\left(M_{P}\right)$, which is the regime most efficiently described in terms of stringy bounces $[11,12]$ rather than by effective theories. For this purpose, it is plausible to investigate how similar physical considerations arise in a class of bouncing models characterized by the contribution of a nonlocal dilaton potential [11]. A related set of solutions can be realized in the context of double field theory [12], which aims at realizing $T$ duality explicitly at the level of component fields of closed string field theory. In this class of bouncing universes [11], the evolution equations in the Einstein frame are

$$
\begin{gathered}
6 \mathcal{H}_{e}^{2}=\frac{\varphi^{\prime 2}}{2}+e^{\varphi} a_{e}^{2} V, \\
4 \mathcal{H}_{e}^{\prime}+2 \mathcal{H}_{e}^{2}=-\left(\frac{\varphi^{\prime 2}}{2}-e^{\varphi} a_{e}^{2} V\right)-e^{\varphi} a_{e}^{2} \frac{\partial V}{\partial \bar{\varphi}}, \\
\varphi^{\prime \prime}+2 \mathcal{H}_{e} \varphi^{\prime}+e^{\varphi} a_{e}^{2}\left(V-\frac{1}{2} \frac{\partial V}{\partial \bar{\varphi}}\right)=0,
\end{gathered}
$$

where, as in Eq. (12), natural gravitational units $16 \pi G=1$ have been adopted. Equations (13)-(15) have been derived in Ref. [11] and can be found, exactly in this form, in the first paper of Ref. [11]. The potential appearing in Eqs. (13)-(15) depends on the $T$-duality-invariant combination $\bar{\varphi}$, namely, $V=V(\bar{\varphi})$, where, ${ }^{9}$ in four space-time dimensions, $\bar{\varphi}=\varphi-3 \ln a_{s}$. Since the potential depends on a $T$-duality-invariant combination, the corresponding results can also be interpreted in a double field theory context (see, in particular, the last two papers of Ref. [12]).

There are various classes of solutions compatible with a violation of the null energy condition in a finite time interval, and some of them, with their own virtues and their potential drawbacks, have been discussed in the past (see, e.g., Ref. [11] and references therein). For the sake of concreteness, we can consider the following potential:

\footnotetext{
${ }^{8}$ This statement follows from Eq. (12). Unless $\epsilon$ is of order 1 (but smaller than 1), the expression on the right-hand side of Eq. (12) is negative.

${ }^{9}$ Note that $a_{s}$ is the scale factor in the string frame, which is related to the scale factor in the Einstein frame as $a_{s}=a_{e} e^{\varphi / 2}$; see the first paper of Ref. [11] for further details.
}

PHYSICAL REVIEW D 96, 101302(R) (2017)

$$
V(\bar{\varphi})=V_{1} e^{2\left(\bar{\varphi}-\bar{\varphi}_{1}\right) / \alpha}\left[1-e^{2\left(\bar{\varphi}-\bar{\varphi}_{1}\right) / \alpha}\right] .
$$

After repeated combinations of Eqs. (13), (14), and (15), an explicit differential relation only involving $\mathcal{H}_{e}$ and $\varphi^{\prime}$ can be derived:

$$
\frac{\partial}{\partial \tau}\left(\varphi^{\prime}+2 \mathcal{H}_{e}\right)+2 \mathcal{H}_{e}\left(\varphi^{\prime}+2 \mathcal{H}_{e}\right)=0
$$

Using now Eq. (17), the bouncing solution corresponding to the potential (16) is given by

$$
a_{e}(\tau)=a_{1}\left[x^{2}+1\right]^{\alpha / 4}, \quad \varphi=\bar{\varphi}_{1}-\frac{\alpha}{2} \ln \left(x^{2}+1\right), \quad x=\frac{\tau}{\tau_{1}},
$$

where, as in Eq. (8), $\tau_{1}$ denotes the typical scale of the bounce and $\mathcal{H}=a_{1} H_{1}=1 / \tau_{1}$. In the rescaled coordinate $x$, the bouncing region corresponds to $|x|<\sqrt{2 /(\alpha+2)}$, and to satisfy consistently all the equations, the relation between the constants $\tau_{1}, V_{1}$, and $\bar{\varphi}_{1}$ must be given by $\tau_{1} a_{1} \sqrt{V_{1}}=\alpha e^{-\varphi_{1} / 2}$ in natural gravitational units. The scale factor (18) generalizes the one of Eq. (8), and the energy density and pressure, in the Einstein frame description, are

$\rho_{e}=\frac{\varphi^{\prime 2}}{2 a_{e}^{2}}+e^{\varphi} V, \quad p_{e}=\frac{\varphi^{\prime 2}}{2 a_{e}^{2}}-e^{\varphi} V+e^{\varphi} \frac{\partial V}{\partial \bar{\varphi}}$.

In terms of $\rho_{e}$ and $p_{e}$, Eq. (15) becomes $\rho_{e}^{\prime}+$ $3 \mathcal{H}_{e}\left(\rho_{e}+p_{e}\right)=0$, as expected; finally, the enthalpy density is

$$
4\left(\mathcal{H}_{e}^{2}-\mathcal{H}_{e}^{\prime}\right)=\varphi^{\prime 2}+e^{\varphi} a_{e}^{2} \frac{\partial V}{\partial \bar{\varphi}}
$$

Even if the evolution Eqs. (13)-(15) are local in time, the nonlocality of the potential that depends on the shifted dilaton $\bar{\varphi}$ is reflected in a substantially different form of the equations that cannot be mimicked ${ }^{10}$ by a potential term depending only on $\varphi$.

As emphasized above, gravitational particle production at the end of inflation $[9,10]$ is caused by the presence of nonconformally coupled scalar fields. While in the general theory of relativity the kinetic term of the gauge fields is conformally coupled to the background, in the case of stringy bounces, gauge bosons can also be amplified thanks to the dilaton coupling; the quantum fluctuations of gauge fields will then contribute to the total energy density (see the third paper of Ref. [11]). In this case, particle production roughly contributes to the total energy density as

\footnotetext{
${ }^{10}$ It is actually well known that if the potential only depends on $\varphi$ [e.g., $W=W(\varphi)$ ] we would simply have $4\left(\mathcal{H}_{e}^{2}-\mathcal{H}_{e}^{\prime}\right)=\varphi^{\prime 2}$ [11].
} 
$\mathcal{N}_{g} H_{1}^{4}\left(a_{1} / a\right)^{4}$, where, in analogy with Eq. (5), $\mathcal{N}_{g}=N_{g} \zeta_{g}$; direct estimates of the evolution of the gauge mode functions suggest that $\zeta_{g}=\mathcal{O}(0.2)[11]$ even if this value is largely irrelevant for the present considerations. This effect has been already studied (both analytically and numerically) in a related context and for a slightly different background (see the third paper of Ref. [11]); in that case, it has been argued that particle production could heat up a cold bounce solution and, simultaneously, stabilize the dilaton. We are now ready to compute the averaged null energy condition in the case of the solution (18). More specifically, the integral of the total enthalpy density becomes

$$
\begin{aligned}
\int_{-\infty}^{+\infty}\left(p_{t}+\rho_{t}\right) d \tau= & \frac{1}{4 \tau_{1} a_{1}^{2}}\left\{\int_{-\infty}^{\tau_{*}} \frac{\alpha\left[(\alpha+2) x^{2}-2\right]}{\left(x^{2}+1\right)^{2+\alpha / 2}} d \tau\right. \\
& \left.+\frac{16}{9} \mathcal{N}_{g} \tau_{*} a_{1} \frac{H_{*}^{4}}{H_{1}}\right\} .
\end{aligned}
$$

In the case of Eq. (21), the analog of Eq. (11) is given by

$$
\left(\frac{\tau_{1}}{\tau_{*}}\right)=\left[\frac{\mathcal{N}_{g}}{6} H_{1}^{2}\right]^{1 /(2-\alpha)}=\epsilon .
$$

Using Eq. (22), we can rephrase Eq. (21) as follows:

$$
\int_{-\infty}^{+\infty}\left(p_{t}+\rho_{t}\right) d \tau=\frac{H_{1}}{a_{1}}\left\{\int_{-\infty}^{\epsilon} \frac{\alpha\left[(\alpha+2) x^{2}-2\right]}{\left(x^{2}+1\right)^{2+\alpha / 2}} d x+\frac{8}{3} \epsilon^{\alpha+5}\right\} .
$$

The integral of Eq. (23) can be performed explicitly in terms of hypergeometric functions so that the averaged null energy condition evaluates to a function of $\alpha$ and $\epsilon$ that can be numerically studied. The averaged null energy condition is positive, provided $0<\alpha<2$ and $0.5<\epsilon<1$. Consequently, as anticipated, the averaged null energy condition is not violated, provided the maximal scale of the bounce is of the order of (but smaller than) the Planck or string scale.

If all energy conditions are enforced throughout the dynamical evolution, nonsingular bouncing universes and their descendants must be largely excluded. In this paper, a less radical and somehow more conservative approach has been explored by suggesting a modest compromise: the null energy condition, even if locally violated, may be satisfied in an averaged sense. According to this prospect, it has been assumed that the null energy condition is violated for a limited amount of time but not in the asymptotic regions (i.e., away from the bounce). Whenever the maximal expansion rate reached by the bounce is of the order of Planck (or string) scales, the null energy condition is more likely to be satisfied in an averaged sense. Conversely, in a heuristic perspective, the enforcement of the (averaged) null energy condition furnishes an independent criterion for the determination of the maximal scale of the bounce.

\section{ACKNOWLEDGEMENT}

It is a pleasure to acknowledge A. Gentil-Beccot and S. Rohr of the CERN Scientific Information Service for their kind assistance.
[1] D. N. Spergel et al., Astrophys. J. Suppl. Ser. 148, 175 (2003); 170, 377 (2007); L. Page et al., Astrophys. J. Suppl. Ser. 170, 335 (2007); B. Gold et al., Astrophys. J. Suppl. Ser. 192, 15 (2011); D. Larson et al., Astrophys. J. Suppl. Ser. 192, 16 (2011); C. L. Bennett et al., Astrophys. J. Suppl. Ser. 192, 17 (2011); G. Hinshaw et al., Astrophys. J. Suppl. Ser. 208, 19 (2013); C. L. Bennett et al., Astrophys. J. Suppl. Ser. 208, 20 (2013); P. A. R. Ade et al., Astron. Astrophys. 571, A22 (2014); 571, A16 (2014); 594, A20 (2016).

[2] J. c. Hwang and H. Noh, Phys. Rev. D 65, 124010 (2002); Phys. Lett. B 545, 207 (2002); J. c. Hwang, Phys. Rev. D 65, 063514 (2002); J. c. Hwang and H. Noh, Phys. Rev. D 71, 063536 (2005); H. S. Kim and J. c. Hwang, Phys. Rev. D 75, 043501 (2007).

[3] M. Novello and S. E. P. Bergliaffa, Phys. Rep. 463, 127 (2008); A. Ashtekar and P. Singh, Classical Quantum Gravity 28, 213001 (2011); S. Nojiri, S. D. Odintsov, and V. K. Oikonomou, Phys. Rep. 692, 1 (2017).
[4] S. Hawking and G. F. R. Ellis, The Large Scale Structure of Space-Time (Cambridge University Press, Cambridge, England, 1973).

[5] S. Weinberg, Phys. Rev. D 77, 123541 (2008); E. Elizalde, A. Jacksenaev, S. D. Odintsov, and I. L. Shapiro, Phys. Lett. B 328, 297 (1994); Classical Quantum Gravity 12, 1385 (1995).

[6] V. A. Rubakov, Phys. Usp. 57, 128 (2014); Usp. Fiz. Nauk 184, 137 (2014); M. Libanov, S. Mironov, and V. Rubakov, J. Cosmol. Astropart. Phys. 08 (2016) 037; T. Kobayashi, Phys. Rev. D 94, 043511 (2016); Y. Cai, Y. Wan, H. G. Li, T. Qiu, and Y. S. Piao, J. High Energy Phys. 01 (2017) 090; Y. Cai, H. G. Li, T. Qiu, and Y. S. Piao, Eur. Phys. J. C 77, 369 (2017).

[7] L. H. Ford, Proc. R. Soc. A 364, 227 (1978); F. J. Tipler, Phys. Rev. D 17, 2521 (1978); C. I. Kuo and L. H. Ford, Phys. Rev. D 47, 4510 (1993); L. H. Ford and T. A. Roman, Phys. Rev. D 51, 4277 (1995); 53, 1988 (1996); 53, 5496 (1996); 60, 104018 (1999). 


\section{MASSIMO GIOVANNINI}

[8] L. Parker and S. A. Fulling, Phys. Rev. D 7, 2357 (1973); A. A. Starobinsky, Pis'ma Astron. Zh. 4, 155 (1978) [Sov. Astron. Lett. 4, 82 (1978)].

[9] L. H. Ford, Phys. Rev. D 35, 2955 (1987); M. Giovannini, Phys. Rev. D 58, 083504 (1998); 73, 083505 (2006); E. D. Schiappacasse and L. H. Ford, Phys. Rev. D 94, 084030 (2016).

[10] L. Parker, Nature (London) 261, 20 (1976); B. L. Hu and L. Parker, Phys. Lett. 63A, 217 (1977).

[11] M. Giovannini, Phys. Rev. D 95, 083506 (2017); 70, 103509 (2004); Classical Quantum Gravity 21, 4209
PHYSICAL REVIEW D 96, 101302(R) (2017)

(2004); M. Gasperini, M. Giovannini, and G. Veneziano, Nucl. Phys. B694, 206 (2004); Phys. Lett. B 569, 113 (2003).

[12] W. Siegel, Phys. Rev. D 47, 5453 (1993); 48, 2826 (1993); A. A. Tseytlin, Phys. Lett. B 242, 163 (1990); Nucl. Phys. B350, 395 (1991); M. J. Duff, Nucl. Phys. B335, 610 (1990); O. Hohm, C. Hull, and B. Zwiebach, J. High Energy Phys. 07 (2010) 016; 08 (2010) 008; H. Wu and H. Yang, J. Cosmol. Astropart. Phys. 07 (2014) 024; C. T. Ma and C. M. Shen, Fortschr. Phys. 62, 921 (2014). 